\title{
Ocupaciones humanas en Cabeza de León 1 (Santa Cruz, Argentina): una aproximación desde la tecnología lítica
}

\author{
Daniela Soledad Cañete Mastrángelo \\ Recibido 22 de octubre 2020. Aceptado 02 de febrero 2021
}

\section{RESUMEN}

La costa patagónica ha sido ocupada desde mínimamente el Holoceno medio, pero el modo en que los humanos interactuaron con el espacio y los recursos a lo largo del tiempo en los distintos sectores fue diferente, lo que hace necesario estudiar las particularidades de cada ocupación. Aquí se abordan las actividades tecnológicas que se habrían desarrollado en el sitio Cabeza de León 1, sito en el Parque Nacional Monte León (Santa Cruz, Argentina), a partir del estudio de los materiales líticos allí recuperados. Estos muestran el empleo mayoritario de materias primas locales, la realización de todas las etapas de manufactura in situ, la implementación de estrategias tecnológicas principalmente expeditivas, y también permiten inferir actividades mayoritariamente vinculadas a la captura y procesamiento de recursos.

Palabras clave: Tecnología lítica; Ocupaciones costeras; Patagonia.

\section{Human occupations at Cabeza de León 1 site, Santa Cruz, Argentina: an approach from the lithic technology}

\begin{abstract}
The Patagonian coast has been occupied at least since the Middle Holocene but the way in which humans have interacted with spaces and resources in different areas of the coast has varied. As such, it is important to study the specifics of each occupation. In this paper, the activities that would have taken place at Cabeza de León 1 archaeological site, Monte León National Park, Santa Cruz province, Argentina, are addressed from the study of the recovered lithic assemblages. Results show the use of local raw material, the development of all manufacture stages in situ and the implementation of mainly expedient strategies. It can also be inferred that activities were mostly related to the capture and processing of resources.
\end{abstract}

Keywords: Lithic technology; Coastal occupations; Patagonia.

\section{LAS OCUPACIONES COSTERAS PATAGÓNICAS: ESTADO DE LA CUESTIÓN}

Diversas investigaciones han mostrado que la costa atlántica patagónica y sus recursos fueron usados en una amplia variedad de formas desde, mínimamente, el Holoceno medio (Miotti, 2006; Castro et al. 2007; Orquera y Gómez Otero, 2007; Salemme, Bujalesky y Santiago, 2007; Favier Dubois y Borella, 2011; Cardillo, 2013; Borrero, 2017; Gómez Otero, Schuster y Banegas, 2017, entre muchos otros). Así, los estudios zooarqueológicos realizados en sitios arqueológicos de Río Negro correspondientes al Holoceno medio informan acerca de una actividad pesquera intensiva, lo que durante el Holoceno tardío cambia hacia un consumo importante de fauna terrestre (Favier Dubois y Scartascini, 2012). Esta última también fue la preferida en sectores de la costa norte de Chubut y algunos puntos de la costa norte de Tierra del Fuego durante el mismo período (Borrero et al., 2008; Gómez Otero, 2008).

Daniela Soledad Cañete Mastrángelo. Consejo Nacional de Investigaciones Científicas y Técnicas (CONICET). Instituto Nacional de Antropología y Pensamiento Latinoamericano (INAPL). 3 de Febrero 1378 (CI46BJN), Ciudad Autónoma de Buenos Aires. E-mail: danielasol.cm@gmail.com

Intersecciones en Antropología 22(1), enero-junio: 111-123. 2021. ISSN-e 1850-373X

https://doi.org/10.37176/iea.22.1.2021.597

Facultad de Ciencias Sociales - UNICEN - Argentina 
Sin embargo, en la costa sur de Chubut y de Santa Cruz, habría predominado la explotación de fauna marina. $Y$, en momentos finales del Holoceno tardío, en la costa norte de Santa Cruz se observa la misma tendencia (Arrigoni, Andrieu y Bañados, 2008; Mansur, 2008; Cruz, Muñoz y Lobbia, 2010; Zilio, 2017).

En cuanto a la tecnología, los conjuntos líticos de la costa patagónica indican una tendencia general en el aprovechamiento de rocas locales, pero estos son diversos, dado que también hay diferencias en las actividades que se realizaron en los distintos sectores y en el uso del espacio, lo que generó sitios con mayor densidad (reflejo de ocupaciones reiteradas y/o prolongadas) y otros con menor densidad artefactual (Arrigoni et al., 2008; Borrero et al., 2008; Gómez Otero, 2008; Cardillo, 2013; Hammond, 2015; Cañete Mastrángelo, 2016 , 2019). Por su parte, la tecnología ósea se encuentra distribuida en varios sectores de la costa (norte y sur de Chubut, norte y sur de Santa Cruz), mientras que la tecnología cerámica se encuentra desde Río Negro hasta la costa norte de Santa Cruz (Arrigoni et al., 2008; Gómez Otero, 2008; Hammond, 2015). Por ello, profundizar acerca de las particularidades de cada sector costero se torna importante a fin de construir conocimiento sobre tales espacios, lo cual a la vez permite ampliar la información disponible sobre las ocupaciones costeras a mayor escala.

En este trabajo se busca aportar información sobre las tareas que llevaron a cabo allí los grupos humanos del pasado y su relación con los recursos locales (como son, por ejemplo, las diversas materias primas) mediante el estudio de la tecnología lítica del sitio arqueológico Cabeza de León 1 (CL1, de aquí en más), que es una de las mayores concentraciones hasta ahora detectadas en el actual territorio del Parque Nacional Monte León (PNML, de aquí en más) (Caracotche et al., 2005). Con ello se busca aportar a un objetivo mayor, que es el de estudiar la relación entre humanos-animalesambiente en la costa sur de Santa Cruz.

La evidencia recuperada hasta el momento en PNML indica que las ocupaciones humanas registrarían una amplia profundidad temporal, que abarca desde los $5550 \pm 90 \mathrm{AP}$ años (correspondiente al sitio Yegua Quemada 3) hasta los $650 \pm 75$ años AP (fechado realizado sobre restos hallados en la zona Restinga norte). Las más tempranas constituyen una de las pocas evidencias de ocupaciones humanas registradas hasta el momento durante el
Holoceno medio en la costa sur de la Patagonia continental, mientras que las más tardías están en concordancia con las detectadas en otros sectores de esta región (Caracotche et al., 2005; Muñoz, Cruz y Caracotche, 2009; Caracotche et al., 2017).

\section{Cabeza de León 1}

Este sitio se encuentra en la localidad arqueológica homónima, caracterizada por poseer amplias concentraciones de material arqueológico. Esta ha sido interpretada como el resultado del uso recurrente durante los últimos 1500 años AP. CL1 se emplaza a $0,13 \mathrm{~km}$ del mar, aproximadamente (Figura 1). Es una de las más grandes y densas dispersiones de materiales conocidas en PNML (Muñoz, 2015, 2019) y ha sido afectada por coleccionistas de material arqueológico hasta que la zona correspondiente al parque se declaró área protegida (Caracotche et al., 2005; Muñoz, 2019).

Los estudios de los conjuntos tecnológicos efectuados en el marco de las investigaciones realizadas para la conformación del parque se limitaron al material recuperado en superficie, el cual consistió en artefactos líticos únicamente. Estos mostraron la predominancia de desechos de talla seguidos por artefactos formatizados, entre los cuales se destacan las raederas. Los núcleos siguen en orden de importancia y se registraron, en baja frecuencia, bola, percutor y molino. Las rocas empleadas para la manufactura son principalmente locales, y hay unas pocas alóctonas (obsidiana verde y negra, ópalo, calcedonia y xilópalo). Llama la atención la presencia de dos núcleos de lutita, pero un solo desecho de talla de esta roca, a la vez que hay varios desechos de talla sobre rocas sin núcleos que se correspondan. Se observaron evidencias de la realización de múltiples actividades tecnológicas a partir de la presencia de varias clases tipológicas de instrumentos y de diversos tipos de desechos de talla (Caracotche et al., 2005).

Posteriormente, se recuperaron materiales estratigráficos que permitieron obtener una datación radiocarbónica (970 \pm 100 años AP, GX-33151), y que aportaron dos muestras zooarqueológicas compuestas por restos de otáridos (Otaria flavescens y Arctocephalus australis), guanaco (Lama guanicoe), ungulados, aves (entre ellas: Phalancrocorax sp. y Rhea sp.), y mamíferos y vertebrados indeterminados, así como roedores que ingresaron por depositación natural (Muñoz, 2015, 2019). El conjunto 


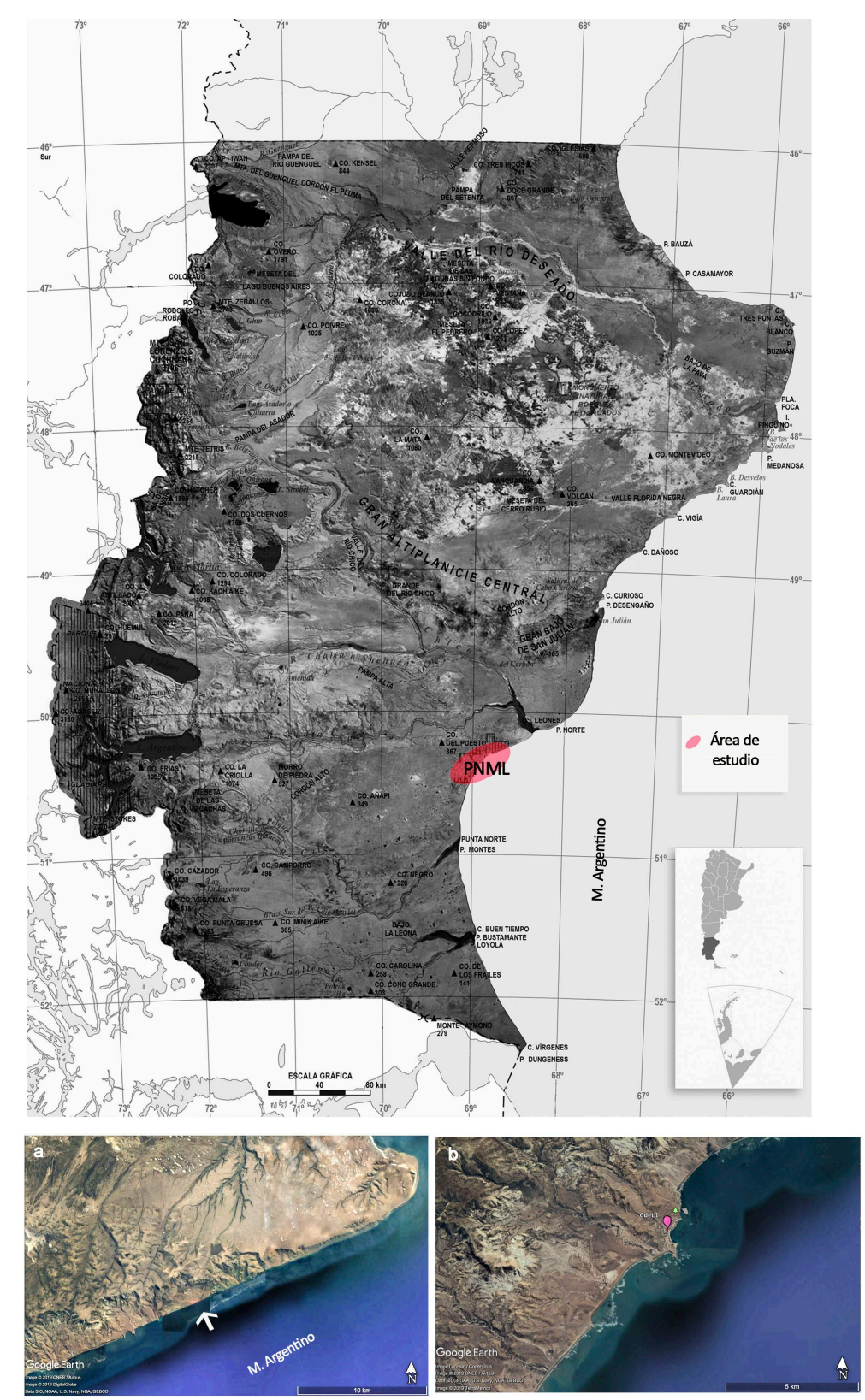

Figura 1. Ubicación de PNML. a) Detalle de la localización de PNML; b) Ubicación de CL1.

animales. Con el análisis aquí presentado se busca ampliar y complementar esta información a fin de obtener una visión más completa sobre los comportamientos de los grupos humanos que habitaron (sensu Ingold, 1993) el área.

\section{LA ORGANIZACIÓN TECNOLÓGICA COMO HERRAMIENTA PARA ESTUDIAR LAS OCUPACIONES COSTERAS}

Esta investigación busca aportar al estudio de diversos aspectos de la vida de las personas y de cómo pudieron haber afectado sus decisiones, el modo en que realizaban las tareas y las consecuencias materiales de ello, por lo cual se eligió como marco teórico la organización tecnológica (sensu Nelson, 1991). Este enfoque tiene como principal objetivo el análisis de la variación producida dentro del registro lítico en la organización de las actividades humanas, que están insertas en determinados patrones ambientales y sociales (Nelson, 1991; Sassaman, 1994; Andrefsky, 2008). Así, permite conectar a la tecnolo-

de restos de pinnípedos es el más importante y está formado por especímenes que, en su mayoría, se encuentran osteológicamente inmaduros. La representación anatómica de otáridos y ungulados sugiere que el conjunto está integrado por varios individuos en cada grupo taxonómico. Las evidencias de procesamiento humano de las carcasas permite postular que las actividades realizadas incluyeron el descarne y desmembramiento de las presas (Muñoz, 2015, 2019).

En conclusión, la evidencia disponible permite plantear que en CL1 se realizaron diferentes actividades que involucraron la manufactura de artefactos líticos y el procesamiento y consumo de gía con aspectos no tecnológicos de la organización humana, ya que aquella es un producto social en el que se condensan diversos aspectos de la sociedad que la creó, por lo que, a la vez, es un reflejo de ella (Gero, 1989; Sassaman, 1994; Colombo y Flegenheimer, 2013, entre otros). La organización tecnológica de un grupo humano puede estudiarse a través del reconocimiento de las estrategias elaboradas, que son los procesos de resolución de situaciones o planes que surgen de la dinámica de interacción entre el ambiente y los seres humanos. Sus consecuencias materiales son las formas de los artefactos y la composición de los conjuntos, y el contexto en el que surgen y la planificación son las variables que inciden sobre el diseño de los 
instrumentos. Estas estrategias no son excluyentes unas de las otras, sino que se ponen en práctica y se complementan de acuerdo con la situación particular que el grupo atraviese (Nelson, 1991; Escola, 2004).

\section{MATERIALES Y MÉTODOS}

En este trabajo fueron analizados los materiales recuperados en estratigrafía $(n=1807)$ de una cuadrícula de $1 \times 1 \mathrm{~m}$ excavada por niveles artificiales de $10 \mathrm{~cm}$ cada uno hasta que se llegó al nivel estéril a los $-30 \mathrm{~cm}$ de profundidad (Figura 2). Las características de los hallazgos se mantuvieron constantes hasta la mencionada profundidad e incluyeron materiales líticos, restos zooarqueológicos y malacológicos. El sedimento que contenía el material arqueológico era arciIloso-arenoso y fue cernido con una zaranda con malla de $2 \mathrm{~mm}$.

El análisis tecnológico siguió la propuesta clasificatoria de Aschero (1975, 1983) y de Aschero y Hocsman (2004) para núcleos, artefactos formatizados y filos naturales con rastros complementarios. En el caso de los desechos de talla, también se incorporó la propuesta de Bellelli, Guráieb y García (1985).

En todas las clases tipológicas se determinó macroscópicamente la materia prima y se registró la reserva de corteza (medida como presencia/ausencia), entendida como un indicador del estadio de

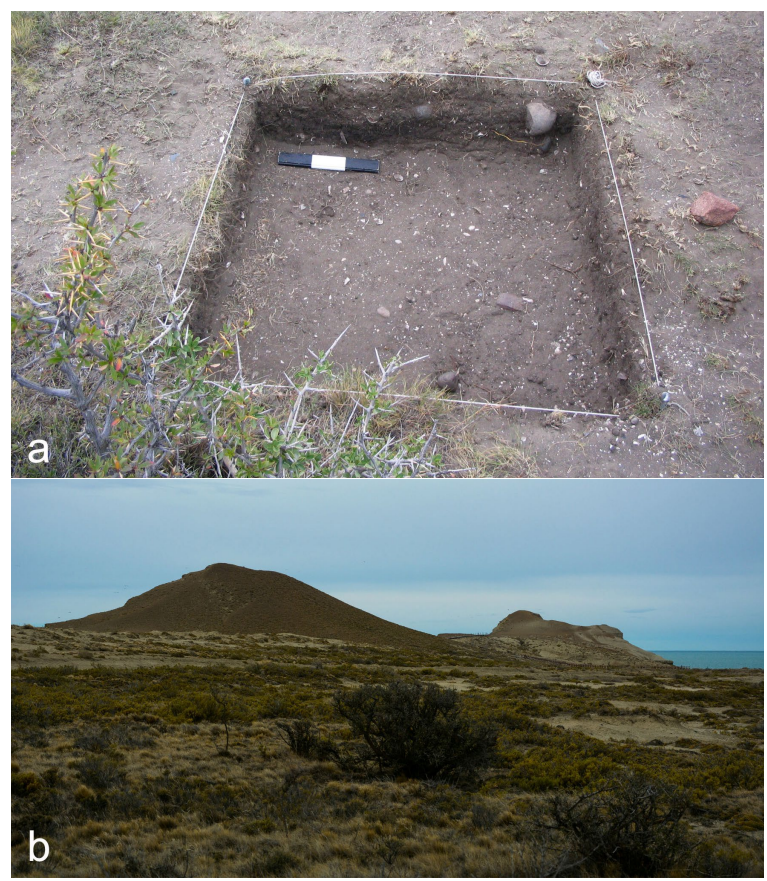

Figura 2. a) Cuadrícula excavada (fotografía: S. Caracotche); b) Vista desde el sitio. talla que representa cada pieza (Bradbury y Carr, 1995; Bellelli, 2005). Además se consignó el estado (entera, fracturada con talón y fracturada sin talón), variable que permitió conocer el número mínimo de piezas que integran cada conjunto.

\section{Núcleos}

Se tomaron las medidas absolutas de las piezas enteras y se consignaron la morfología (forma general), la totalidad de negativos de lascado y de plataformas y los defectos en la manufactura. A partir de estas variables, puede conocerse el estado de explotación que presenta el núcleo, lo que permite definir, en consecuencia, la intensidad del aprovechamiento de las distintas materias primas (Aschero, 1975, 1983; Paulides, 2006).

\section{Desechos de talla}

Se registró el tamaño de aquellas piezas que se conservan enteras. Este surge de la combinación del largo y el ancho de cada una de ellas (para mayor información sobre medidas absolutas y relativas de las piezas de todas las clases tipológicas, ver Cañete Mastrángelo, 2019). Para ello se empleó el gráfico de Bagolini (1968) modificado por Aschero (1975, 1983). Fueron observados, en los desechos enteros y fracturados con talón, el tipo de lasca y el tipo de talón, además de las variables mencionadas previamente. Ello permite conocer los estadios de talla presentes, las conductas tecnológicas desarrolladas (Aschero, 1975, 1983; Bradbury y Carr, 1995, 1999; Guráieb y Espinosa, 1998; Odell, 2004; Bellelli, 2005) y la estrategia seguida para la manufactura de los artefactos (Odell, 2004; Patten, 2009).

\section{Artefactos formatizados}

Se identificaron el tamaño (exclusivamente en piezas enteras), el grupo tipológico; la cantidad de filos por pieza, el ángulo medido, el estado del filo y la serie técnica (anchura y profundidad de los lascados sobre la pieza) (Aschero, 1975, 1983; Aschero y Hocsman, 2004). Estas variables sirvieron para evaluar la intensidad en el aprovechamiento de la materia prima (Shott, 1986; Nelson, 1991; Aschero et al., 1995), la inversión de energía puesta en la manufactura (Escola y Hocsman, 2008; Guráieb, 2012) y el estado en el que fueron descartados, entendiéndose que filos con ángulos superiores a 
los $80^{\circ}$ se encontrarían embotados (Aschero et al., 1995; Escola, 2004).

\section{Filos naturales con rastros complementarios}

Para esta clase tipológica se utilizaron las mismas variables empleadas en el análisis de los artefactos formatizados.

\section{RESULTADOS}

CL1 posee un conjunto lítico que incluye siete núcleos, 1746 desechos de talla, 52 artefactos formatizados y dos piezas asignables a la clase filos naturales con rastros complementarios.

\section{Núcleos $(n=7)$}

Se recuperaron cuatro piezas fracturadas y tres enteras. Se registraron cinco tipos de rocas utilizadas para su manufactura: basalto $(\mathrm{n}=2)$, rocas silíceas $(n=2)$, arenisca $(n=1)$, dacita $(n=1)$ y ópalo $(n=1)$. La amplia mayoría presentan reserva de corteza $(n=6)$. Las medidas absolutas de las piezas enteras son: $41 \times 32 \times 27 \mathrm{~mm}$ (roca silícea), $50 \times 47 \times 35 \mathrm{~mm}$ (arenisca) y otra mucho más pequeña de ópalo $(22 \times 21 \times 10 \mathrm{~mm})$.

En cuanto a la forma general, solo se identificaron dos tipos: discoidal irregular o parcial $(n=5)$ y globulosa $(n=2)$. La cantidad de negativos de lascados es variable entre las piezas: cuatro $(n=2)$, cinco, ocho, nueve y 11 ( $\mathrm{n}=1$ en cada caso). En general, los núcleos presentan solo una plataforma explotada. Las piezas con dos y tres plataformas son una en cada caso.

Se identificaron múltiples defectos de manufactura sobre todos los núcleos, y en la mayoría de los casos, están combinados. Los más frecuentes son las charnelas y las terminaciones quebradas $(n=6$ en cada caso). Se observaron también terminaciones quebradas escalonadas $(n=3)$, golpes fallidos $(n=2)$, charnelas escalonadas $(n=2)$ y frente de extracción astillado $(\mathrm{n}=2)$.

\section{Desechos de talla $(n=1746)$}

La mayor parte se encuentran fracturados. Si se consideran en conjunto los fracturados con talón ( $\mathrm{n}=878,50 \%$ ) y sin talón $(\mathrm{n}=785,45 \%$ ), se reúne el $95 \%$ de la muestra. Las piezas enteras son 83 (5\%). El número mínimo de desechos es 961.

En la Tabla 1 se presentan los tres estados considerados y puede observarse que hay tres materias primas que se destacan por sobre el resto: basalto, dacita y rocas silíceas. Debido a que las fracturadas sin talón pueden sobrerrepresentar la muestra, se consideran solamente las piezas enteras y fracturadas con talón para determinar la cantidad de materias primas presentes en el conjunto.

El tamaño más frecuente es el muy pequeño ( $n=44,53 \%$ ). Los demás tamaños registrados son: pequeño ( $\mathrm{n}=23,28 \%$ ), mediano-pequeño $(\mathrm{n}=6$, $7 \%)$, mediano-grande $(n=4,5 \%)$ y grande $(n=6$, $7 \%)$.

El tipo de lasca más abundante es el angular, que reúne más del $76 \%$ de la muestra. Las segundas en orden de importancia son las planas, seguidas por las de dorso natural. Los productos bipolares, las de flanco de núcleo y de reactivación directa son los tipos menos representados (Tabla 2). Por su parte, la reserva de corteza posee baja frecuencia: solo el $17 \%$ de la muestra presenta esta característica.

\begin{tabular}{|l|c|c|c|c|}
\hline MATERIA PRIMA & ENT & FCT & FST & N \\
\hline Andesita & - & 1 & - & 1 \\
\hline Arenisca & 3 & 13 & 8 & 24 \\
\hline Basalto & 30 & 302 & 300 & 632 \\
\hline Calcedonia & - & 16 & 21 & 37 \\
\hline Cuarcita & 4 & 34 & 28 & 66 \\
\hline Dacita & 11 & 135 & 111 & 257 \\
\hline Granito & - & 1 & - & 1 \\
\hline Indeterminada & - & 9 & 5 & 14 \\
\hline Limolita & - & 4 & 1 & 5 \\
\hline Obsidiana gris & - & 3 & - & 3 \\
\hline Obsidiana gvv & - & 2 & 2 & 4 \\
\hline Obsidiana negra & 1 & 1 & - & 2 \\
\hline Ópalo & - & 6 & 12 & 18 \\
\hline Riolita & - & 3 & 5 & 8 \\
\hline Rocas Silíceas & 31 & 326 & 277 & 634 \\
\hline Toba & 3 & 22 & 15 & 40 \\
\hline Total & 83 & 878 & 785 & 1746 \\
\hline \% & 5 & 50 & 45 & 100 \\
\hline
\end{tabular}

ENT = entero, FCT = fracturado con talón, FST = fracturado sin talón, $\mathrm{N}=$ total, obsidiana gvv = obsidiana gris verdosa veteada.

Tabla 1. Desechos de talla por materia prima y estado: frecuencias absolutas. 


\begin{tabular}{|c|c|c|c|c|c|c|c|c|c|c|c|}
\hline \multirow[b]{2}{*}{ TIPO DE LASCA } & \multicolumn{9}{|c|}{ MATERIAS PRIMAS } & \multirow[b]{2}{*}{$\mathbf{N}$} & \multirow[b]{2}{*}{$\%$} \\
\hline & 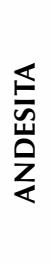 & 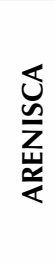 & $\begin{array}{l}\stackrel{0}{E} \\
\frac{1}{\infty} \\
\frac{1}{\infty}\end{array}$ & 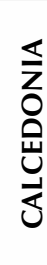 & 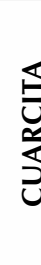 & 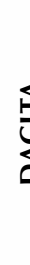 & & 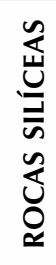 & 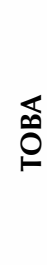 & & \\
\hline Angular & 1 & 11 & 255 & 14 & 34 & 9 & & 275 & 19 & 730 & 76 \\
\hline Arista & & & 5 & & & 2 & & 12 & & 20 & 2,1 \\
\hline Bending & & 1 & 4 & & 1 & & & 11 & 3 & 21 & 2,2 \\
\hline Dorso natural & & 3 & 25 & & 1 & 5 & & 10 & & 46 & 4,8 \\
\hline Flanco de núcleo & & & & & & & & 2 & & 2 & 0,2 \\
\hline Producto bipolar & & & & & & 2 & & 1 & & 4 & 0,4 \\
\hline Plana & & 1 & 14 & 2 & 1 & 2 & & 32 & 2 & 80 & 8,3 \\
\hline Primaria & & & 11 & & 1 & 2 & & 2 & 1 & 18 & 1,9 \\
\hline Reactivación directa & & & & & & & & 1 & & 1 & 0,1 \\
\hline Secundaria & & & 18 & & & 9 & & 11 & & 39 & 4,1 \\
\hline Total & 1 & 16 & 332 & 16 & 38 & 14 & & 357 & 25 & 961 & 100 \\
\hline
\end{tabular}

Tabla 2. Tipos de lasca: frecuencias absolutas y relativas por materia prima.

En este conjunto se destacan los talones lisos $(\mathrm{n}=347,36,1 \%)$ y filiformes $(\mathrm{n}=311,32,4 \%)$. Los segundos y terceros en orden de importancia son los astillados ( $n=97,10,1 \%$ ) y puntiformes $(n=95,9,9 \%)$ respectivamente. Las piezas en las que no se pudo identificar el talón (debido a sus características tecnológicas (por ejemplo, desechos de talla poliédricos) y/o a que su tamaño era muy pequeño y no permitió que se lo viera con claridad) son poco frecuentes $(n=14,1,5 \%)$ (Tabla 3).

\begin{tabular}{|l|c|c|}
\hline \multicolumn{1}{|c|}{ TIPO DE TALÓN } & $\mathbf{N}$ & $\mathbf{\%}$ \\
\hline Astillado & 97 & 10,1 \\
\hline Cortical & 31 & 3,2 \\
\hline Diedro & 36 & 3,7 \\
\hline Facetado & 30 & 3,1 \\
\hline Filiforme & 311 & 32,4 \\
\hline Liso & 347 & 36,1 \\
\hline Puntiforme & 95 & 9,9 \\
\hline Sin identificar & 14 & 1,5 \\
\hline Total & 961 & 100 \\
\hline
\end{tabular}

Tabla 3. Tipos de talón: frecuencias absolutas y relativas.

\section{Artefactos formatizados $(n=52)$}

La mayor parte de la muestra se encuentra fracturada; solo 17 piezas están enteras. En cuanto al tamaño, se destaca el grande $(n=5,29,4 \%)$. A este le siguen: mediano-pequeño $(n=4,23,5 \%)$, pequeño, muy grande $(\mathrm{n}=3,17,6 \%$ en cada caso) y mediano-grandes $(n=2,11,8 \%)$.

Las materias primas más representadas son el basalto y las rocas silíceas $(n=19,36,5 \%$ en ambos casos); y las menos frecuentes son la cuarcita, la obsidiana (de la variedad gris verdosa veteada) y una roca indeterminada $(n=1,1,9 \%$ en cada caso) (Tabla 4). El 55\% de la muestra no presenta reserva de corteza.

Una pequeña parte de la muestra de artefactos formatizados posee más de un filo, lo que explica la diferencia entre la cantidad de piezas $(n=52)$ y de filos $(n=60)$. Se observaron artefactos formatizados con dos $(n=7,12 \%)$ y tres $(n=1,1,7 \%)$ filos, entre los que se observaron las siguientes combinaciones: dos raederas, raedera y muesca, raedera y raspador, tres raspadores, dos raspadores, dos artefactos de formatización sumaria, raspador y raedera y punta entre muescas y raedera.

Se identificaron 12 grupos tipológicos, de los cuales el raspador es el de mayor importancia (tipos: filo frontal corto [ $n=6$ ], filo lateral corto [ $n=4$ ], filo fronto-bilateral $[n=2]$, fragmentos no diferenciados $[n=2]$, filo frontal restringido $[n=1]$ y filo lateral largo $[n=1])$, y la raedera es la segunda en orden de importancia (comprende: filo lateral largo $[n=10]$ y filo frontal largo $[n=3])$. Los grupos 
tipológicos menos representados son el bifaz y la muesca (Tabla 4, Figuras 3 y 4 ).

En cuanto al estado de los filos, se observó que la amplia mayoría se encuentra embotado ( $n=44$, $91,7 \%)$. Solo cuatro fueron descartados activos $(\mathrm{n}=8,3 \%)$. Para este cálculo no se contabilizaron las puntas de proyectil ni los esbozos de pieza bifacial, por tal motivo, el n total es de 50 filos. Esta decisión

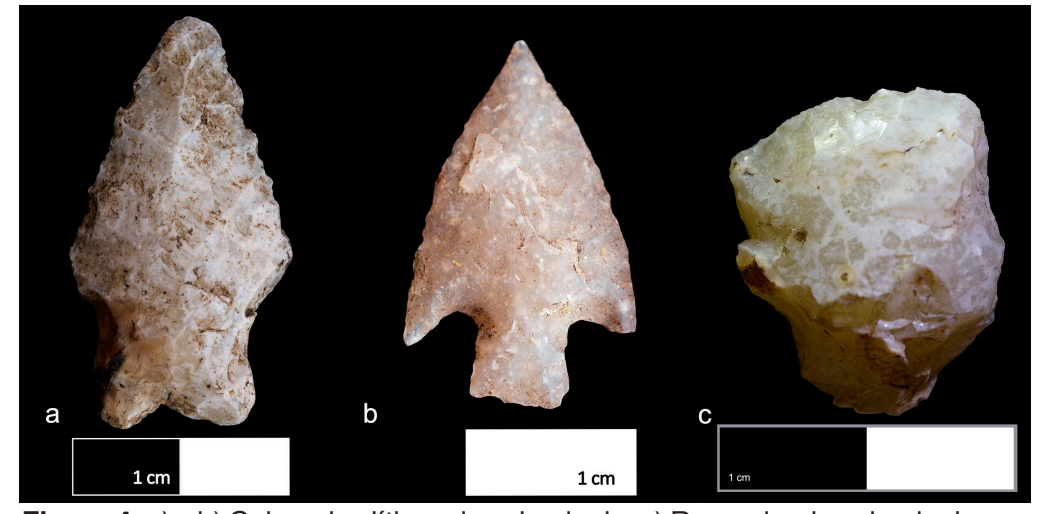

Figura 4. a) y b) Cabezales líticos de calcedonia; c) Raspador de calcedonia. se basa en que se asume que la actividad en la que se emplearon las primeras no habría generado desgaste del filo (el contacto entre la presa y los filos es muy corto como para generar rastros sobre los últimos [Rots y Plisson, 2014]) y los esbozos de pieza bifacial no son un artefacto formatizado terminado, por lo que la medición de los ángulos de sus filos no sería confiable.

Entre la anchura de los lascados se registró retoque $(n=50)$, microrretoque $(n=34)$ y retalla $(n=26)$. Debe tenerse en cuenta que estos pueden presentarse combinados en un mismo filo, lo cual explica que la suma del $\mathrm{n}$ de cada uno supere el total de filos contabilizados. La observación de la extensión de los lascados sobre la cara de las piezas mostró filos marginales $(\mathrm{n}=39$ ), totalmente extendidos ( $n=13$, esperable por la presencia de piezas bifaciales) y parcialmente extendidos $(n=6)$. Por último, se identificó una mayoría de filos unifaciales $(n=43)$, mientras que la cantidad de filos bifaciales asciende a 17.

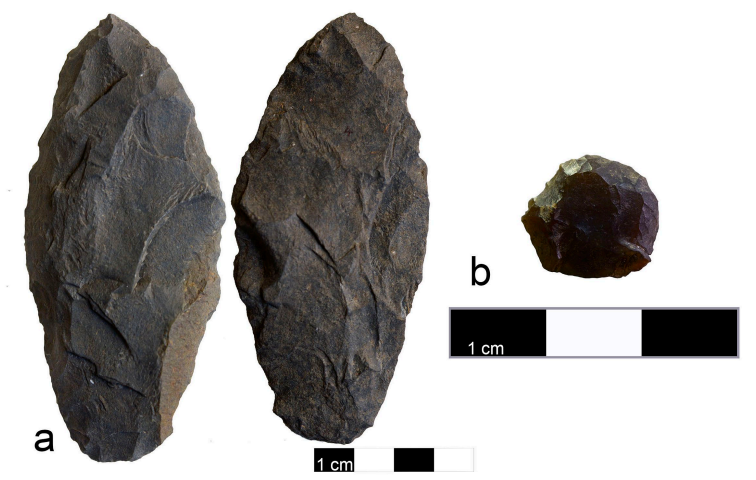

Figura 3. a) Raedera de basalto; b) Raspador de roca silícea (xilópalo).

\section{Filos naturales con rastros complementarios $(n=4)$}

Se observaron cuatro filos naturales con rastros complementarios, dos de los cuales constituyen el

\begin{tabular}{|c|c|c|c|c|c|c|c|c|}
\hline \multirow[b]{2}{*}{ FILOS } & \multicolumn{8}{|c|}{ MATERIA PRIMA } \\
\hline & 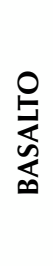 & 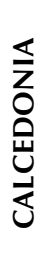 & 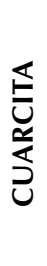 & $\frac{\mathbb{E}}{\mathrm{E}}$ & 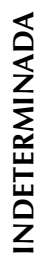 & 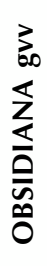 & $\begin{array}{l}\frac{n}{u} \\
\underline{\underline{U}} \\
\overline{\bar{n}} \\
\dot{\alpha}\end{array}$ & $\stackrel{\Sigma}{\varrho}$ \\
\hline Bifaz & 1 & - & - & - & - & - & - & 1 \\
\hline Raspador & 5 & 4 & - & - & 2 & - & 5 & 16 \\
\hline Raedera & 4 & - & 1 & 4 & - & - & 4 & 13 \\
\hline Muesca & - & - & - & 1 & - & - & - & 1 \\
\hline Pta. e/muescas & - & - & - & 1 & - & - & 1 & 2 \\
\hline Perforador & 2 & - & - & 1 & - & - & 1 & 4 \\
\hline Pta. Proyectil & 3 & 2 & - & - & - & - & 3 & 8 \\
\hline Art. Form. Sum. & 1 & - & - & 1 & - & 1 & 6 & 9 \\
\hline Esbozo P. bif. & 3 & - & - & - & - & - & - & 3 \\
\hline Fgto. no dif. & 1 & - & - & - & - & - & 2 & 3 \\
\hline Total & 20 & 6 & 1 & 8 & 2 & 1 & 22 & 60 \\
\hline
\end{tabular}

Pta. e/muescas $=$ punta entre muescas, Pta. Proyectil $=$ punta de proyectil, Art. Form. Sum. = artefacto de formatización sumaria, Esbozo P. bif. = esbozo de pieza bifacial, Fgto. no dif. = fragmento no diferenciado, OBSIDIANA gvv = obsidiana gris verdosa veteada, R. SILÍCEAS = rocas silíceas.

Tabla 4. Grupos tipológicos identificados en las distintas materias primas: frecuencias absolutas.

único filo de la pieza y los otros dos se encuentran combinados con una raedera y un artefacto de formatización sumaria en la misma forma base. Los dos primeros fueron confeccionados sobre cuarcita y dacita, y los segundos, sobre dacita y basalto. La única pieza entera es de tamaño grande. Todos estos filos se encuentran embotados.

\section{Resumen de resultados obtenidos en el conjunto de superficie y excavación}

A fin de brindar un panorama más completo acerca del conjunto lítico de CL1, en la Tabla 5 se 


\begin{tabular}{|l|c|c|}
\hline CLASE ARTEFACTUAL & $\begin{array}{c}\text { SUPERFICIE } \\
(\mathbf{N})\end{array}$ & $\begin{array}{c}\text { EXCAVACIÓN } \\
(\mathbf{N})\end{array}$ \\
\hline Bifaz & 1 & 1 \\
\hline Núcleo & 14 & 7 \\
\hline Punta de proyectil & 3 & 8 \\
\hline Raedera & 12 & 13 \\
\hline Raspador & 3 & 16 \\
\hline Molino & 1 & - \\
\hline Fragmento de artefacto formatizado & 1 & 3 \\
\hline Percutor & 1 & - \\
\hline Desechos de talla & 302 & 1746 \\
\hline Muesca & - & 1 \\
\hline Punta entre muescas & - & 2 \\
\hline Perforador & - & 1 \\
\hline Bola & 1 & - \\
\hline Artefacto de formatización sumaria & 2 & 9 \\
\hline Esbozo pieza bifacial & - & 3 \\
\hline Filos naturales con rastros \\
complementarios & - & 4814 \\
\hline Total & 341 & 13 \\
\hline
\end{tabular}

Tabla 5. Clases artefactuales de los conjuntos de superficie y estratigrafía: frecuencias absolutas.

presenta un resumen de las diferentes clases artefactuales que integran el registro de superficie y el de excavación. Los datos del primero fueron obtenidos de Caracotche y colaboradores (2005).

En ambos contextos predominan los desechos de talla, pero se observa que el conjunto de excavación posee mayor diversidad artefactual, lo que es esperable por tratarse de un conjunto mejor preservado de agentes tafonómicos naturales y culturales.

\section{DISCUSIÓN}

El registro lítico de CL1 lo diferencia de otros sitios estudiados en el área y de la localidad vecina Punta Entrada (Cañete Mastrángelo, 2016, 2019; Caracotche et al., 2017), ya que posee la mayor cantidad de artefactos formatizados registrada hasta el momento. Sin embargo, el registro zooarqueológico se corresponde con las evidencias recuperadas en otros sitios de la localidad. Se destacan los restos de pinnípedos, que en su mayoría serían individuos inmaduros, con lo cual se repite el patrón de la predominancia de estos animales, seguidos por ungulados.
El conjunto artefactual indica que en CL1 se llevaron a cabo todas las etapas de la reducción lítica, debido a que se recuperaron núcleos, desechos de talla de varios tipos con diversidad de talones y artefactos formatizados en diferentes estadios de su trayectoria de vida. Asimismo, se recuperaron piezas con y sin reserva de corteza ( $17 \%$ y $83 \%$ respectivamente), las que aportan a estas interpretaciones. Se registra una correspondencia entre la mayor parte de las materias primas que integran cada clase artefactual, a saber, entre las empleadas en la manufactura de los núcleos y desechos de talla y entre aquellas utilizadas en estos últimos, los artefactos formatizados y los filos naturales con rastros complementarios. Las rocas que se encuentran en todas las clases artefactuales son las rocas silíceas, el basalto y la dacita, las que a la vez son las más representadas en el conjunto y de disponibilidad local (ver Cañete Mastrángelo, 2019). En cuanto a los tamaños, es posible hacer una observación similar, ya que desechos de talla y artefactos formatizados se presentan en todas las categorías de tamaño, por lo que habría una paridad entre estos últimos y potenciales formas base, como así también entre los lascados que conforman los filos y los desechos de talla. Los núcleos seguirían esta tendencia pero, como la mayor parte de ellos se encuentra fracturado, no es posible hacer mayores inferencias. Los talones de los desechos de talla apoyan estas observaciones, dado que se registraron tipos relacionados con las primeras etapas de la reducción (por ejemplo, corticales), junto con otros vinculados a estadios más avanzados (por ejemplo, facetados [Bradbury y Carr, 1999]). Los talones lisos pueden vincularse a la confección de los filos (Espinosa, 1995), mientras que los filiformes y puntiformes serían el resultado de la talla por presión, una de las técnicas empleadas en el mantenimiento de los filos de los instrumentos (Espinosa, 1995).

El conjunto de artefactos formatizados está compuesto por 60 filos e incluye una amplia variedad de grupos tipológicos. La mayor parte de ellos se encuentra embotado, lo que se tomó como indicador de uso (sensu Aschero, 1975, 1983), dado que hasta el momento no pudieron realizarse análisis traceológicos. Los filos identificados (gran cantidad de raspadores y raederas) sugerirían la realización 
de diversas tareas con cierto énfasis en el procesamiento de los recursos bióticos, especialmente animales y sus productos derivados. Esto deberá verificarse en un futuro con estudios funcionales. Las raederas fueron asociadas en otros sectores de la Patagonia con la realización de diversas tareas, como son cortar, raspar y perforar (Álvarez et al., 2010), por lo que podrían interpretarse como diseños versátiles (sensu Nelson, 1991), mientras que los raspadores se habrían usado para tareas de corte y raspado (por ejemplo, Álvarez, 2004; Cueto, 2013). Esto guarda relación con las huellas antrópicas encontradas en los restos faunísticos de CL1 (Muñoz, 2015, 2019). Asimismo, la presencia de varios cabezales líticos se vincularía con la captura de diversas presas (otáridos y ungulados), tal como fue propuesto por Cañete Mastrángelo (2019). Teniendo en cuenta la presencia de esbozos de piezas bifaciales, el uso de materias primas locales para su manufactura y la presencia de múltiples desechos de talla con tamaños coincidentes con diferentes estadios de la reducción lítica, es posible que en CL1 se hayan confeccionado cabezales líticos. El análisis Ilevado a cabo en Cañete Mastrángelo (2019) mostró que el conjunto de cabezales líticos de PNML estaría integrado por diferentes sistemas de armas, que podrían asociarse a presas de diversos portes y comportamientos. CL1 es de relevancia para esta observación por ser el sitio en el que, hasta el momento, se recuperó la mayor cantidad de este tipo de piezas juntas $(n=8)$.

Las materias primas utilizadas son, en su mayoría, de origen local e inmediatamente disponibles (sensu Civalero y Franco, 2003), y entre ellas se destacan el basalto, las rocas silíceas y la dacita. También se registraron rocas alóctonas: calcedonia, ópalo, obsidianas gris, gris verdosa veteada y negra (en el conjunto de superficie también se identificó obsidiana verde [Caracotche et al., 2005]). Todas estas rocas fueron observadas entre los desechos de talla. La calcedonia y la obsidiana gris verdosa veteada también fueron utilizadas para manufacturar raspadores y cabezales líticos (la obsidiana solamente para estos últimos en el conjunto de superficie [Caracotche et al., 2005]). El ópalo, además, se identificó entre los núcleos. Hasta el momento se presume que la calcedonia y el ópalo son alóctonos, ya que no se los ha observado en las prospecciones realizadas en el área ni se mencionan en la bibliografía especializada sobre el tema (Martínez y Kutschker, 2011). Respecto de las obsidianas, se conoce que la variedad negra es de la fuente de Pampa del Asador; la gris, del volcán
Chaitén; y la gris verdosa veteada, del Cordón de Baguales, según lo informan los estudios geoquímicos realizados (Cruz et al., 2011; Stern et al., 2012). Todas provienen de puntos cardinales diferentes y de distancias de entre $300 \mathrm{~km}$ hasta $1000 \mathrm{~km}$ lineales desde el área de estudio, lo que permite postular la posible existencia de un vínculo entre los grupos de cazadores-recolectores que estuvieron presentes en este sector de la costa con aquellos que habrían circulado por otros puntos de la Patagonia, o que sean los mismos grupos que se trasladaban por el espacio.

Merece señalarse que la tendencia observada entre los artefactos de superficie es semejante a la de la muestra aquí analizada. En ambos casos predominan los desechos de talla, seguidos por artefactos formatizados y luego núcleos. Asimismo, en los dos conjuntos hay desechos de talla de materias primas que no fueron identificadas entre los núcleos. Caracotche y colaboradores (2005) plantean que la acción de coleccionistas habría desencadenado en esta situación. Sin embargo, podrían plantearse explicaciones alternativas. Podrían existir núcleos sobre los que se trabajaba en CL1 pero que luego eran transportados a otros puntos del espacio teniendo como contrapartida otros núcleos cuyos productos fueran los que se trasladaran. Por otra parte, los procesos de formación de sitio podrían ser una causa que explique esta diferencia, ya que las piezas más pequeñas se enterrarían con mayor facilidad, y dejarían sobrerrepresentadas en superficie aquellas de mayores tamaños, como los núcleos, tal como fue observado por Borrazzo (2009) en otros contextos costeros. Sumado a ello, la acción de los fuertes vientos de la zona promovería la pérdida de los tamaños más pequeños del conjunto superficial (Borrazzo, 2009). Sin embargo, esta última explicación no daría cuenta de la diferencia entre materias primas de desechos de talla y núcleos hallados en excavación. Lo más plausible es que los tres factores hayan contribuido. La disponibilidad y accesibilidad de materias primas en el área, los múltiples defectos de manufactura registrados sobre los núcleos recuperados (y que podrían ser la causa de abandono de estos) y la ausencia de reclamación no se corresponderían con explicaciones alternativas como por ejemplo, el equipamiento del espacio.

En conclusión, puede decirse que, en general, la tecnología lítica presenta tendencias hacia el uso de estrategias expeditivas a partir de la preponderancia de la talla de artefactos unifaciales, la presencia 
de filos marginales, el empleo de materias primas locales, el uso de diseños versátiles, la talla in situ y el manejo poco económico de la materia prima -reflejado en los múltiples defectos de manufactura en núcleos-, el descarte de piezas con tamaños que posibilitarían la reactivación de sus filos y, potencialmente, el uso de filos naturales (Nelson, 1991; Aschero et al., 1995; Wallace y Shea, 2006). Por otra parte, las rocas que componen el conjunto tecnológico también permitieron observar vínculos con otros espacios de la región patagónica, los que pudieron involucrar la circulación de bienes y/o de individuos, lo que muestra que este sector de la costa formaba parte de amplios rangos de acción y circulación de personas.

En CL1 se habrían desarrollado diversos tipos de tareas, entre las que puede mencionarse el procesamiento y consumo de animales (Muñoz, 2015, 2019), la talla y (posible) uso de artefactos líticos $y$, potencialmente, el procesamiento de otro tipo de materiales (por ejemplo, cuero, huesos para la manufactura de artefactos, madera, entre otros), como lo sugiere la diversidad de grupos tipológicos entre los artefactos formatizados. La presencia mayoritaria de filos agotados apoyaría la idea de que allí no solo se habrían confeccionado los artefactos formatizados, sino que también se utilizaran y/o se haya hecho recambio de toolkit. Así, puede plantearse que el tiempo que los grupos humanos pasaban en este espacio era suficiente como para poder realizar todas estas actividades, por lo que no se trataría de estancias breves, sino prolongadas y/u ocupaciones redundantes, tal como lo sugiere Lobbia (2012) para otro sitio de PNML. Una situación semejante fue descripta para la costa norte de Santa Cruz por Zubimendi, Castro y Moreno (2004) y Zilio (2017).

Evidencias recuperadas en ciertos puntos de PNML muestran la existencia de ocupaciones desde el Holoceno medio (Caracotche et al., 2017), lo que implica una importante profundidad temporal en el contacto de los grupos humanos con este sector de la costa santacruceña. La evidencia disponible para dicho periodo señala que ya se explotaban las materias primas locales y las mismas obsidianas identificadas en CL1. De igual modo, los recursos bióticos elegidos fueron los mismos que se seleccionaron durante el Holoceno tardío (Caracotche et al., 2017). A partir de ello, es posible plantear la existencia de un conocimiento del área y de los recursos que esta ofrecía y una continuidad en el modo de interactuar con dichos recursos a lo largo del tiempo. Con el fin de seguir indagando en las relaciones entre ambiente, animales y humanos en el pasado, será necesario profundizar el estudio de múltiples líneas de evidencia, las que en conjunto brindarán mayor información sobre el modo en que los humanos incorporaban el espacio costero en sus vidas.

\section{Agradecimientos}

A los Dres. Sebastián Muñoz y Mariana Carballido. Se contó con financiamiento del CONICET (PIP CONICET 996 y 0359), UNPA 29/ A364 y UNC 33820180100101CB. Al personal de APN, por su colaboraron con las tareas logísticas y de excavación.

\section{REFERENCIAS CITADAS}

Álvarez, M. (2004). El uso de materias primas vegetales en la costa norte del Canal de Beagle a través del análisis funcional de base microscópica. En T. Civalero, P. Fernández y G. Guráieb (Eds.), Contra viento y Marea. Arqueología de la Patagonia (pp. 279-294). Sociedad Argentina de Antropología e Instituto Nacional de Antropología y Pensamiento Latinoamericano.

Álvarez, M., Britz, I., Pal, N. y Salvatelli, L. (2010). Contextos de uso y diseños: una propuesta metodológica para el análisis de la variabilidad de los conjuntos líticos. En R. Bárcena y $\mathrm{H}$. Chiavazza (Ed.), Actas del XII Congreso Nacional de Arqueología Argentina, tomo I/V (pp. 67- 72). Facultad de Filosofía y Letras UNCUYO. Instituto de Ciencias Humanas, Sociales y Ambientales, CONICET.

Andrefsky Jr., W. (Ed.) (2008). An introduction to stone tool life history and technological organization. En Lithic technology (pp. 3-22). Cambridge University Press.

Arrigoni, G., Andrieu, M. y Bañados, C. (2008). Arqueología de cazadores-recolectores prehistóricos en la costa central del golfo San Jorge. En I. Cruz y S. Caracotche (Eds.), Arqueología de la costa patagónica. Perspectivas para la conservación (pp. 91-108). Universidad Nacional de la Patagonia Austral.

Aschero, C. (1975). Ensayo para una clasificación morfológica de los artefactos líticos aplicada a estudios tipológicos comparativos. Informe al CONICET. MS.

Aschero, C. (1983). Ensayo para una clasificación morfológica de los artefactos líticos aplicada a estudios tipológicos comparativos. Informe al CONICET. Revisión. MS 
Aschero, C. y Hocsman, S. (2004). Revisando cuestiones tipológicas en torno a la clasificación de artefactos bifaciales. En D. Loponte, A. Acosta y M. Ramos (Comps.), Temas de Arqueología. Análisis lítico (pp. 7-26). Talleres gráficos del Departamento de Publicaciones e Imprenta, Secretaría de Extensión Universitaria de la Universidad Nacional de Luján.

Aschero, C., Moya, L., Sotelos, C. y Martínez, J.

(1995). Producción lítica en los límites del bosque cordillerano: el sitio Campo Río Roble 1 (Santa Cruz, Argentina). Relaciones de la Sociedad de Antropología, XX, 205-238.

Bagolini, B. (1968) Ricerche sulle dimensioni dei manufatti litici preistorici non ritoccati. Annali dell' Università di Ferrara XV: Paleontologia Umana e Paletnologia, 10, 195-219.

Bellelli, C. (2005). Tecnología y materias primas a la sombra de Don Segundo. Una Cantera-taller en el valle de Piedra Parada. Intersecciones en Antropología, 6, 75-92.

Bellelli, C., Guráieb, G. y García, J. (1985). Propuesta para el análisis y procesamiento por computadora de desechos de talla lítica (DELCO-desechos líticos computarizados). Arqueología Contemporánea, II(1), 36-56.

Borrazo, K. (2009). El uso prehistórico de los afloramientos terciarios en la bahía San Sebastián (Tierra del Fuego, Argentina). En M. Salemme, F. Santiago, M. Álvarez, E. Piana, M. Vázquez y M. Mansur (Eds.), Arqueología de Patagonia: Una mirada desde el último confín (pp. 291-305). Utopías.

Borrero, L. A. (2017). Forests, steppes, and coastlines: zooarchaeology and the prehistoric exploitation of Patagonian habitats. En U. Albarella, H. Russ, K. Vickers y S. Viner-Daniels (Eds.), The Oxford Handbook of Zooarchaeology (pp. 674-687). Oxford University Press.

Borrero, L. A., Martín, F., Horwitz, V., Franco, N., Favier Dubois, C., Borella, F.,... y Borrazo, K. (2008). Arqueología de la costa norte de Tierra del Fuego. En I. Cruz y S. Caracotche (Eds.), Arqueología de la costa patagónica. Perspectivas para la conservación (pp. 205265). Universidad Nacional de la Patagonia Austral.

Bradbury, A. y Carr, P. (1995). Flake typologies and alternative approaches: an experimental assesment. Lithic Technology, 20, 100-115.

Bradbury, A. y Carr, P. (1999). Examining stage and continuum models of flake debris analysis: an experimental approach. Journal of Archaeological Science, 26, 105-116.
Cañete Mastrángelo, D. (2016). Estrategias tecnológicas en la desembocadura del Río Santa Cruz, Patagonia Meridional, Argentina. Magallania, 44(1), 211-228.

Cañete Mastrángelo, D. (2019). Decisiones tecnológicas, técnicas de caza y uso del espacio en la Patagonia Meridional. Aportes desde la tecnología lítica de Punta Entrada y Parque Nacional Monte León (tesis doctoral). Universidad de Buenos Aires, Buenos Aires, Argentina.

Caracotche, S., Cruz, I., Espinosa, S., Carballo Marina, F. y Belardi, J. B. (2005). Rescate arqueológico en el Parque Nacional Monte León (Santa Cruz, Argentina). Magallania, 33(2), 143-163.

Caracotche, S., Muñoz, A. S., Cañete Mastrángelo, D. y Lobbia, P. (2017). Yegua Quemada 3: un depósito arqueológico del Holoceno medio en el Parque Nacional Monte León (Patagonia, Argentina). Magallania, 45(2), 165-180.

Cardillo, M. (2013). Cambios en el paisaje, uso del espacio y conjuntos líticos promediados en la costa norte del Golfo San Matías (Río Negro, Argentina) durante el Holoceno medio-tardío. Comechingonia virtual, 1, $1-26$.

Castro, A., Zubimendi, M. A., Andolfo, M. A., Videla, B., Ambrústolo, P., Mazzitelli, L. y Bogan, S. (2007). Cronología de la ocupación humana en la Costa Norte de Santa Cruz: Actualización de datos radiocarbónicos. En F. Morillo, M. Martinic, A. Prieto y G. Bahamonde (Eds.), Arqueología de Fuego-Patagonia. Levantando piedras, desenterrando huesos... y develando arcanos (pp. 527-539). Ediciones CEQUA.

Civalero, T. y Franco, N. (2003). Early human occupations in western Santa Cruz province, southernmost South America. Quaternary International, 109-110, 77-86.

Colombo, M. y Flegenheimer, N. (2013). La elección de rocas de colores por los pobladores tempranos de la Región Pampeana (Buenos Aires, Argentina). Nuevas consideraciones desde las canteras. Boletín del Museo Chileno de Arte Precolombino, 18(1), 125-137.

Cruz, I., Muñoz, A. S. y P. Lobbia (2010). Zooarqueología al sur del río Santa Cruz (Patagonia argentina). Los restos de fauna de P 96 (Punta Entrada) y CL 1 (P. N. Monte León). En J. Bárcena y H. Chiavazza (Eds.) Arqueología Argentina en el Bicentenario de la Revolución de Mayo (Actas del XVII Congreso Nacional De Arqueología Argentina) (pp. 315-320). Universidad Nacional de Cuyo.

Cruz, I., Caracotche, S., Stern, C., Muñoz, A. S., Suby, J., Lobbia, P.,... y Cañete Mastrángelo, D. (2011). Obsidianas y otros indicadores de circulación y uso del espacio en Punta Entrada y P. N. Monte León (Santa 
Cruz, Argentina). En Libro de resúmenes VIII Jornadas de Arqueología de la Patagonia. Museo de Historia Natural de San Rafael.

Cueto, M. (2013). Estudio comparativo forma-función de artefactos líticos. Evidencias de las ocupaciones iniciales de la localidad arqueológica La María. En A. Zangrando, R. Barberena, A. Gil, G. Neme, M. Giardina, L. Luna, C. Otaola, L. Paulides, L. Salgán y A. Tivoli (Eds.), Tendencias teórico metodológicas y casos de estudio en la arqueología Patagónica (pp. 325-334). Museo de Historia Natural de San Rafael, Sociedad Argentina de Antropología e Instituto Nacional de Antropología y Pensamiento Latinoamericano.

Escola, P. (2004). La expeditividad y el registro arqueológico. Chungara Volumen especial, 1, 49-60.

Escola, P. y Hocsman, S. (2008). Inversión de trabajo y diseño en contextos líticos agro-pastoriles (Antofagasta de la Sierra, Catamarca). Cuadernos del Instituto Nacional de Antropología y Pensamiento Latinoamericano, 21, 75-90.

Espinosa, S. (1995). Dr. Scholl y Monsieur Fleur: de talones y bulbos. Cuadernos del Instituto Nacional de Antropología y Pensamiento Latinoamericano, 16, 315-327.

Favier Dubois, C. y Borella F. (2011). Contrastes en la costa del golfo: una aproximación al estudio del uso humano del litoral rionegrino en el pasado. En F. Borella y M. Cardillo (Comps.), Arqueología de pescadores y marisqueadores en Nordpatagonia. Descifrando un registro de más de 6000 años (pp. 13-42). Dunken.

Favier Dubois, C. y Scartarscini, F. (2012). Intensive fishery scenarios on the North Patagonian coast (Río Negro, Argentina) during the Mid-Holocene. Quaternary International, 256, 62-70.

Gero, J. (1989). Assessing social information in material objects: how well do lithics measure up? En R. Torrence (Ed.), Time, energy and stone tools (pp. 92105). Cambridge University Press.

Gómez Otero, J. (2008). Arqueología de la costa centroseptentrional de Patagonia Argentina. En I. Cruz y S. Caracotche (Eds.), Arqueología de la costa patagónica. Perspectivas para la conservación (pp. 72-81). Universidad Nacional de la Patagonia Austral.

Gómez Otero, J., Schuster, V. y Banegas, A. (2017). Archaeology of the Península Valdés: spatial and temporal variability in the human use of the landscape and geological resources. En P. Bouza y A. Bilmes (Eds.), Late Cenozoic of Península Valdés, Patagonia, Argentina. An interdisciplinary approach (pp. 233-262). Springer.
Guráieb, G. (2012). Tendencias tecnológicas, de selección de materias primas y diseño de artefactos líticos en la secuencia de ocupaciones del Holoceno Tardío de Cerro de los Indios 1 (Cl1), lago Posadas, provincia de Santa Cruz (tesis doctoral). Universidad de Buenos Aires, Buenos Aires, Argentina.

Guráieb, G. y Espinosa, S. (1998). La secuencia de producción lítica del alero Dirección Obligatoria: algunas dimensiones del problema. Actas y Memorias del XI Congreso Nacional de Arqueología Argentina ( $8^{a}$ parte). Revista del Museo de Historia Natural de San Rafael, XX(1/4), 159-171.

Hammond, H. (2015). Sitios concheros en la costa norte de Santa Cruz: su estructura arqueológica y variabilidad espacial en cazadores recolectores patagónicos (tesis doctoral). Universidad Nacional de La Plata, La Plata, Argentina.

Ingold, T. (1993). The temporality of landscape. World Archaeology, 25(2), 152-174.

Lobbia, P. (2012). Escrlerocronología en valvas de Mytilus Spp: análisis del sitio $\mathrm{CCH} 4$ (Parque Nacional Monte León, Santa Cruz, Argentina) e implicaciones para la arqueología de Patagonia. Magallania, 40(2), 221-231.

Mansur, M. E. (2008). Arqueología de la zona de Punta Bustamante (Prov. de Santa Cruz, Argentina). En I. Cruz. y S. Caracotche (Eds.), Arqueología de la costa patagónica. Perspectivas para la conservación (pp. 173194). Universidad Nacional de la Patagonia Austral.

Martínez, O. y Kutschker, A. (2011). The "Rodados Patagónicos" (Patagonian Shingle Formation) of eastern Patagonia: environmental conditions of gravel sedimentation. Biological Journal of the Linnean Society, 103, 336-345.

Miotti, L. (2006). La fachada atlántica, como puerta de ingreso alternativa de la colonización humana de América del Sur durante la transición Pleistoceno/ Holoceno. En J. Jiménez López (Ed.), $2^{\circ}$ Simposio Internacional del Hombre Temprano en América (pp. 155-188). Instituto Nacional de Antropología e Historia.

Muñoz, A. S. (2015). El registro zooarqueológico del Parque Nacional Monte León (Santa Cruz, Argentina): una perspectiva desde el sitio arqueológico Cabeza de León 1. Arqueología, 21(2), 261-276.

Muñoz, A. S. (2019). Nuevos datos zooarqueológicos para el sitio arqueológico Cabeza de León. Parque Nacional Monte León (Patagonia Argentina). Cuadernos del Instituto Nacional de Antropología y Pensamiento Latinoamericano. Series Especiales, 7(2), 201-209.

Muñoz, A. S., Cruz, I. y Caracotche, S. (2009). Cronología de la costa al sur del río Santa Cruz: Nuevas dataciones radiocarbónicas en Punta Entrada y Parque Nacional Monte León (provincia de Santa Cruz, Argentina). Magallania, 37(1), 19-38. 
Nelson, M. (1991). The study of technological organization. Archaeological Method and Theory, 3, 57-100.

Odell, G. (2004). Lithic analysis. University of Tulsa.

Orquera, L. y Gómez Otero, J. (2007). Los cazadoresrecolectores de las costas de Pampa, Patagonia y Tierra del Fuego. Relaciones de la Sociedad Argentina de Antropología, XXXII, 75-99.

Patten, B. (2009). Old tools-New eyes, a primal primer of flintknapping. Stone Dagger Publications.

Paulides, L. (2006). El núcleo de la cuestión. El análisis de los núcleos en los conjuntos líticos. En C. Pérez de Micou (Ed.), El modo de hacer las cosas: artefactos y ecofactos en arqueología (pp. 67-100). Universidad de Buenos Aires.

Rots, V. y Plisson, H. (2014). Projectiles and the abuse of the use-wear method in a search for impact. Journal of Archaeological Science, 48, 154-165.

Salemme, M., Bujalesky, G. y Santiago, F. (2007). La Arcillosa 2: la ocupación humana durante el Holoceno medio en el río Chico, Tierra del Fuego, Argentina. En F. Morello, M. Martinic, A. Prieto y G. Bahamonde (Comps.), Arqueología de Fuego-Patagonia: levantando piedras, desenterrando huesos... y develando arcanos (pp. 723-739). Ediciones CEQUA.
Sassaman, K. (1994). Changing strategies of biface production in South Carolina Coastal Plain. En P. Carr (Ed.), The Organization of North American prehistoric chipped stone tool technologies. Archaeological Series 7 (pp. 99-117). International Monographs in Prehistory.

Shott, M. (1986). Technological organization and settlement mobility: an ethnographic examination. Journal of Anthropological Research, 42(1), 15-52.

Stern, C., Caracotche, S., Cruz, I. y Charlin, J. (2012). Obsidiana gris porfírica calcoalcalina del volcán Chaitén en sitios arqueológicos al sur del río Santa Cruz, Patagonia Meridional. Magallania, 40(1), 137-144.

Wallace, I. y Shea, J. (2006). Mobility patterns and core technologies in the Middle Paleolithic of the Levant. Journal of Archaeological Science, 33, 1293-1309.

Zilio, L. (2017). La dinámica humana en la costa norte de Santa Cruz durante el Holoceno tardío: evidencias desde el registro mortuorio e isotópico. Relaciones de la Sociedad Argentina de Antropología, XLII(2), 305-331.

Zubimendi, M., Castro, A. y Moreno, E. (2004). Una aproximación hacia la definición de modelos de uso de la costa norte de Santa Cruz. Magallania, 32, 209-220. 
